\title{
X-ray streak camera temporal resolution improvement using a longitudinal time-dependent field *
}

\author{
J. Qiang, ${ }^{1}$ J. M. Byrd, J. Feng, G. Huang \\ Lawrence Berkeley National Laboratory, Berkeley, California 94720
}

\begin{abstract}
X-ray streak cameras (XSC) have been known to be one of the fastest detectors for ultrafast X-ray science. A number of applications in material science, biochemistry, accelerator physics, require sub-picosecond resolution to study new phenomena. In this paper, we report on a new method which can potentially improve the temporal resolution of a streak camera down to 100 femtoseconds. This method uses a time-dependent acceleration field to lengthen the photoelectron bunch, significantly improving the time resolution as well as reducing the time dispersion caused by initial energy spread and the effects from the space charge forces. A computer simulation of an XSC using this method shows significant improvement in the resolution.
\end{abstract}

Key words: X-ray streak camera, longitudinal magnification

\section{Introduction}

Ultra-fast spectroscopy with X-ray streak cameras provides an important tool for study of dynamics in a number of areas such as the laser plasma interaction, photo reaction in material science and biochemistry, and magnetization. A number of studies have been reported on improving the temporal resolution of X-ray streak camera below 1 ps [1-5]. However, it becomes more and more difficulty to build an X-ray streak camera with temporal resolution below 500 fs due to limits of time dispersion from the energy spread of photo electrons, space-charge effects among photo electrons, finite static image size

\footnotetext{
* This work was supported by the U.S. Department of Energy under Contract No. DE-AC02-05CH11231.

1 Corresponding author: jqiang@lbl.gov
} 
and deflection sweeping speed, and time jitter if multiple shot accumulation operation mode is used. Among these factors, the energy spread of photo electrons presents an intrinsic limit to the temporal resolution of X-ray streak camera [3,5]. This paper describes a novel technique to improve the resolution of XSC by magnifying the longitudinal structure of the electron pulse. This is done by applying a time-dependent electric field along the direction of motion of the electrons and allowing temporal dispersion to lengthen the pulse, decreasing space charge effects and effectively increasing the capability of the subsequent transverse deflection.

A schematic diagram of a prototype XSC at Advanced Light Source (ALS) of Lawrence Berkeley National Laboratory is shown in Fig. 1 [6]. It consists

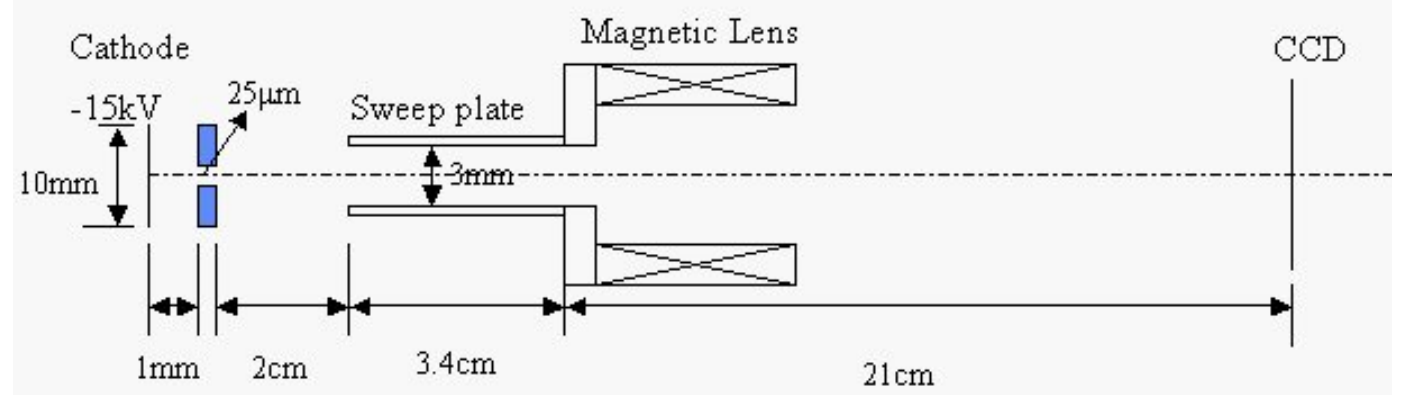

Fig. 1. A schematic plot of a typical X-ray streak camera.

of a photocathode, a longitudinal acceleration anode, a transverse deflection device, a magnetic focusing element, and a CCD image screen. X-rays incident on a photocathode create a bunch of photoelectrons which is accelerated in a static or dynamic field. The electron bunch is subjected to a transverse deflection and focussed on a screen. The temporal resolution of the XSC can be expressed as several error sources in quadrature (under a first-order approximation) given by [7]

$$
\tau=\sqrt{t_{j}^{2}+t_{s w}^{2}+t_{e}^{2}+t_{a d}^{2}+t_{s c}^{2}}
$$

Here, $t_{j}$ is the temporal resolution contributed by the time jitter. It can be reduced by improving photoconductive switch design and by improving laser stability. Very low timing jitter has been achieved in recent studies $[5,8]$. For single shot operation mode, this term disappears. The $t_{s w}$ is the temporal resolution limited by the static image width and the finite vertical deflection speed:

$$
t_{s w}=L w / v_{s}
$$


where $L w$ is the full width half maximum (FWHM) of the vertical static image and $v_{s}$ is the sweeping speed of deflection plate. The size of the static image $L w$ depends on the transverse spatial resolution on the image plane. The sweeping speed of the deflection plates depends on the rise time of photoconductive switch, the field amplitude across the plates, and the longitudinal speed of the electron beam. The time dispersion $t_{e}$ through the acceleration region is given by $[1]$ :

$$
t_{e}=\frac{2.63 \sqrt{\delta E}}{E_{z}}(p s)
$$

where $\delta E$ is the FWHM of the photo electron energy distribution in $\mathrm{eV}$, and $E_{z}$ is the external acceleration field in $k V / m m$. The energy distribution of the photo electrons depends on the material properties of the photocathode and the incident photon energy. For an X-ray incident on metalic photocathode, the photo electrons have a broad energy distribution up to a few electron volts [9]. The external acceleration field is limited by the field-emission effects between the cathode and the anode. Besides the time dispersion between the cathode and the anode, there is another time dispersion factor $t_{a d}$ contributing to the limit of temporal resolution due to the drift between the anode and the deflection plane. Given the energy spread, the distance between the photo cathode and the sweeping plate, this term is given by:

$$
t_{a d}=\frac{L_{d} \delta E}{m c^{3} \gamma_{0}^{3} \beta_{0}^{3}}
$$

where $L_{d}$ is the distance between the anode and the deflection plate, $m$ is the electron mass, $c$ is the speed of light in the vacuum, $\gamma_{0}=1 / \sqrt{1-\beta_{0}^{2}}, \beta_{0}=v_{0} / c$, $v_{0}$ is the average speed of the electron bunch. The last term $t_{s c}$ in Eq. 1 represents the temporal resolution limit from the space-charge broadening. For an intense X-ray light incident on the photo cathode, the space-charge forces among the photo electrons will further elongate the beam and cause extra energy spread. The effects of space-charge depend on the number of electrons in a single shot, the spatial distribution of the electrons, the energy of electrons, and the traveling distance of the electron bunch.

The energy spread of photo electrons presents an intrinsic limit to the temporal resolution of X-ray streak camera. In a streak camera, the temporal information of an incident X-ray light is converted into longitudinal information of photo electrons based on photo-electric effects. Due to the initial energy spread, electrons with different longitudinal positions will mix with each other during the transport to the deflection plate. The mixing of electrons before the deflection plate will destroy the signal imbedded in the photo electrons from the incident light. The deflection plate will no longer be able 
to convert the longitudinal temporal information into transverse spatial information. This causes loss of information and defines a temporal resolution that can be achieved. To reduce the extent of mixing before the deflection plate, one way is to reduce the transport time by making electron higher energy and by shortening the distance between the cathode and the deflection plate. However, the electron energy is determined by the acceleration voltage. It can not be set as arbitrarily high before field breakdown. The typical constant acceleration voltage is about several $10 \mathrm{~s} \mathrm{kV}$. A very high electron beam energy also reduces the efficiency of deflection plate. Another way to reduce the longitudinal mixing of electrons is to make the relative longitudinal positions of electrons larger so that the electrons will reach the deflection plate before they mix. The temporal information imbedded inside the electron beam can then be converted into spatial signal through a time-dependent vertical deflection. In this paper, we report on a method to increase the relative longitudinal separations of photo electrons by using a time-dependent longitudinal field.

\section{Longitudinal Amplification of Photo Electron Bunch in Time- Dependent Field}

The longitudinal relative positions of electrons inside a bunch can be magnified by passing through a time-dependent accelerating field. By appropriately choosing field ramping direction and phase of the beam with respect to the field, the electron arriving earlier see a larger accelerating field than the electron arriving later. This results in a separation between the early electron and the later electron and produces a longitudinal magnification of electron bunch.

The longitudinal equations of motion for an electron inside a time-dependent field without space-charge forces are given by:

$$
\begin{aligned}
\frac{d \Delta t}{d z} & =-\frac{\Delta E}{m c^{3} \gamma_{0}^{3} \beta_{0}^{3}} \\
\frac{d \Delta E}{d z} & =q\left(E_{z}\left(z, \Delta t+t_{0}\right)-E_{z}\left(z, t_{0}\right)\right)
\end{aligned}
$$

where, $\Delta t=t-t_{0}$ is the time difference between an electron and the assumed reference electron, $t_{0}$ is the time of flight of the reference electron, $t$ is the time of flight of the electron, $\Delta E=E-E_{0}$ is the energy difference between the electron and the reference electron, $E_{0}$ is the kinetic energy of the reference electron, $E$ is the kinetic energy of the electron, and $E_{z}$ is the external electrical field. Here, the reference electron can be visualized as the centroid of the photo electrons, the $\Delta t$ is related to the relative longitudinal position of an electron

inside the beam. In above equations, we neglect the transverse variation of the 
field. Assuming that $\Delta t$ is small compared with the time scale of the electric field, Eq. 5 stays the same. The linerized equations of motion for Eq. 6 is:

$$
\frac{d \Delta E}{d z}=q E_{z}^{\prime} \Delta t
$$

where the superscript prime denotes derivative with respect to time. For a linear time-dependent electric field, $E_{z}^{\prime}$ is a constant. For longitudinal magnification, the $q E_{z}^{\prime}$ needs to less than zero. Neglecting energy change of the reference electron, the time difference $\Delta t$ after a distance L will be:

$$
\Delta t_{L}=\cosh (k L) \Delta t_{0}-\frac{k}{q E_{z}^{\prime}} \sinh (k L) \Delta E_{0}
$$

where $k=\sqrt{\left|q E_{z}^{\prime} /\left(m c^{3} \gamma_{0}^{3} \beta_{0}^{3}\right)\right|}$.

\section{Example: ALS X-ray streak camera}

As an illustration, we have used a prototype streak camera [6] at the ALS to numerically test above concept. It consists of a photocathode made of gold with assumed $4.6 \mathrm{eV}$ work function for X-ray light, an acceleration region with $15 \mathrm{kV}$ voltage, a meander sweeping plate for vertical deflection, a solenoid magnetic focusing lens, and a CCD image detector. The field near the axis inside the deflection plate is assumed to be an ideal traveling wave with only vertical component $E_{y}$ :

$$
E_{y}(z, t)=\frac{E_{y 0}}{v_{0} t_{r}}\left(\left(z-z_{0}\right)-v_{0}\left(t-t_{0}\right)\right)
$$

where $E_{y 0}$ is the deflection field strength, $t_{r}$ is the linear rise time, $v_{0}$ is the longitudinal propagation speed of the wave, $z_{0}$ is the starting location of the deflection plate, and $t_{0}$ is the starting time of the sweeping. Here, we assume that the wave propagating inside the meander is well matched to the electron beam velocity. The deflection field is $1.67 \times 10^{5} \mathrm{~V} / \mathrm{m}$ with $100 \mathrm{ps}$ rise time. The photo electrons emitted from the gold cathode has a $\cos (\theta)$ angular distribution, where $\theta$ is the angle with respect to the normal of the cathode surface. The energy distribution of the photo electrons has a form [9]:

$$
N(E) \propto \frac{E}{\left(E+W_{f}\right)^{4}}
$$


where $W_{f}$ is work function of the cathode. The transverse spatial distribution is assumed to be uniformly distributed inside a rectangular box with a size of $100 \mathrm{um}$ in horizontal direction and $25 \mathrm{um}$ in vertical direction. In this example, the longitudinal distribution is two 100 fs square wave laser pulses with adjustable temporal separation. Fig. 2 shows the transverse rms size of photo electrons inside the streak camera without and with vertical streak for an initial 1000 fs separation of two pulses. It is seen that the beam of photo
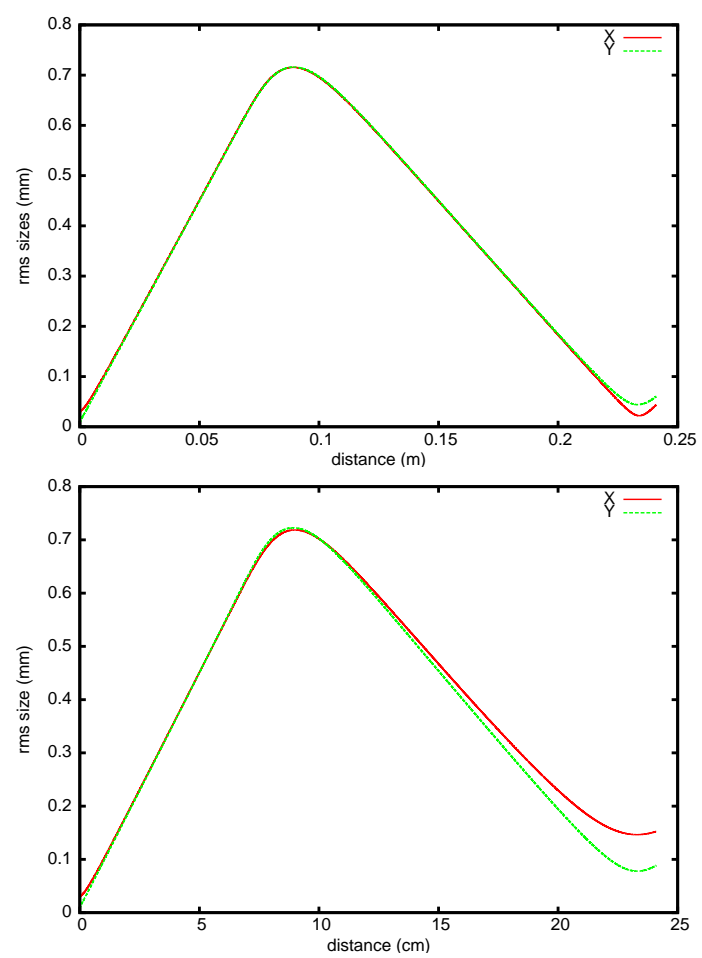

Fig. 2. Transverse rms size of the beam without (top) and with (bottom) streak as a function of distance inside the streak camera.

electrons is focused down to a minimum size near the CCD image plane. This helps to reduce the static image line width contribution to the temporal resolution. The vertical magnification factor for the static image is about 2 . With the vertical sweeping turned on, the transverse image size becomes larger on the CCD screen due to including the conversion of longitudinal separation of two pulses into transverse size by the time-dependent vertical deflection.

Fig. 3 shows the initial longitudinal-transverse distribution of photo electrons and the final transverse distribution of photo electrons on the CCD screen. The top panel is the initial longitudinal positions of the two X-ray pulses. The bottom panel is the streaked image on the CCD screen. The initial longitudinal separation of two pulses is 1000 fs. Through the streak camera, the two pulses are well separated on the transverse plane. This generates two clear-cut streaks on the CCD image plane.

Fig. 4 shows the line density distribution at the CCD image plane with initial 

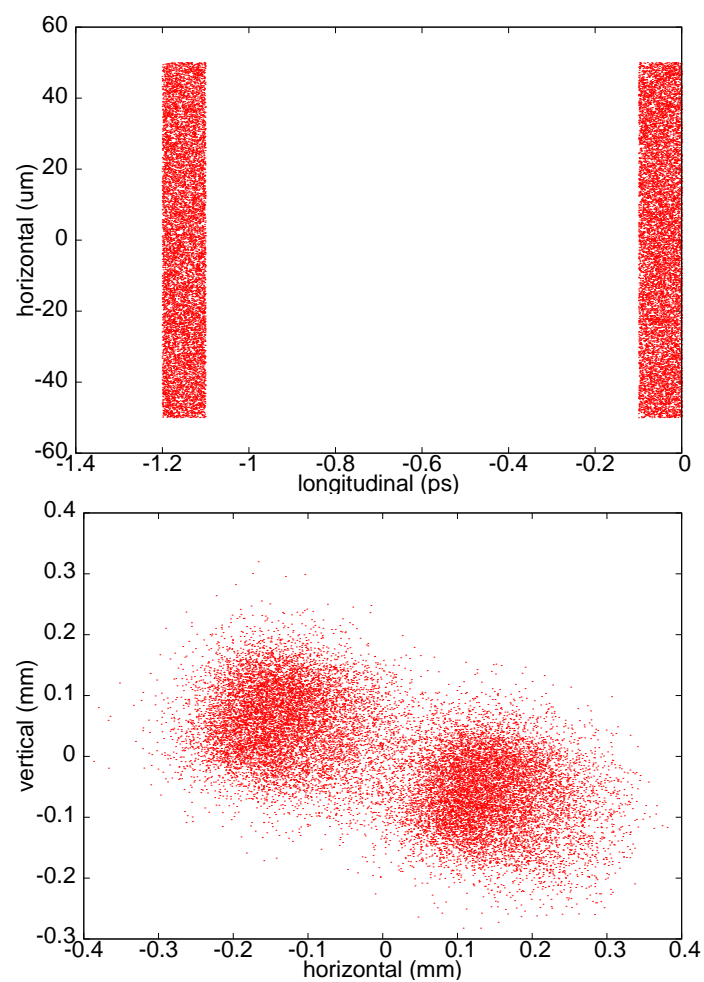

Fig. 3. Initial horizontal-longitudinal distribution of photo electrons and the final transverse distribution of photo electrons on the CCD screen.

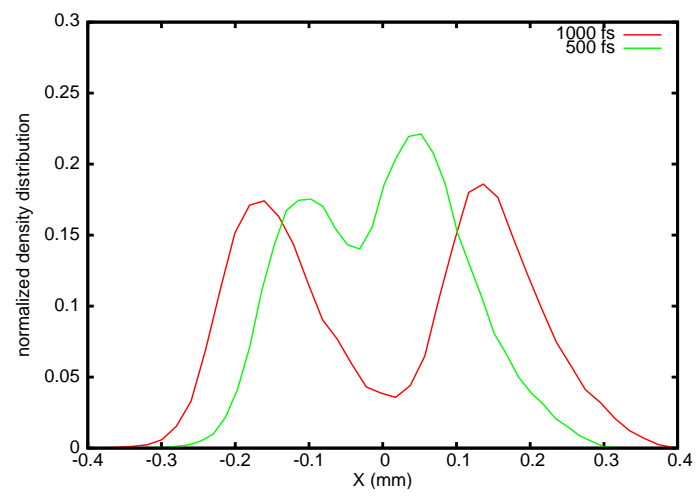

Fig. 4. Line density distribution at the image plane with initial 1000 fs separation and $500 \mathrm{fs}$ separation through the proposed ALS streak camera.

1000 fs separation of two pulses and 500 fs separation in above prototype streak camera. It can be seen that with 1000 fs initial temporal separation, the two pulses can be well distinguished on the transverse image plane. With the temporal separation down to $500 \mathrm{fs}$, the two pulses on the image plane is hardly distinguishable and the resolution power is reduced.

To check the concept of longitudinal magnification, we assume a time-dependent accelerating field inside the acceleration region. The field is linearly ramped from initial $19.25 \mathrm{kV}$ down to $12.62 \mathrm{kV}$ during 26.54 ps time flight of electron inside the gap. Fig. 5 shows the line density distribution with initial $330 \mathrm{fs}$ sep- 
aration of the two pulses without and with longitudinal magnification through the time-dependent field. Without using the longitudinal time-dependent field, the photo electrons from two light pulses get so much mixed that after the vertical deflection, there is no way to separate out these two pulses on the image plane. The temporal information from the X-ray light imbedded inside the initial photo electrons is lost. With the longitudinal time-dependent field, it is seen that the two pulses are still well separated on the image plane even for 330 fs separation of two pulses.
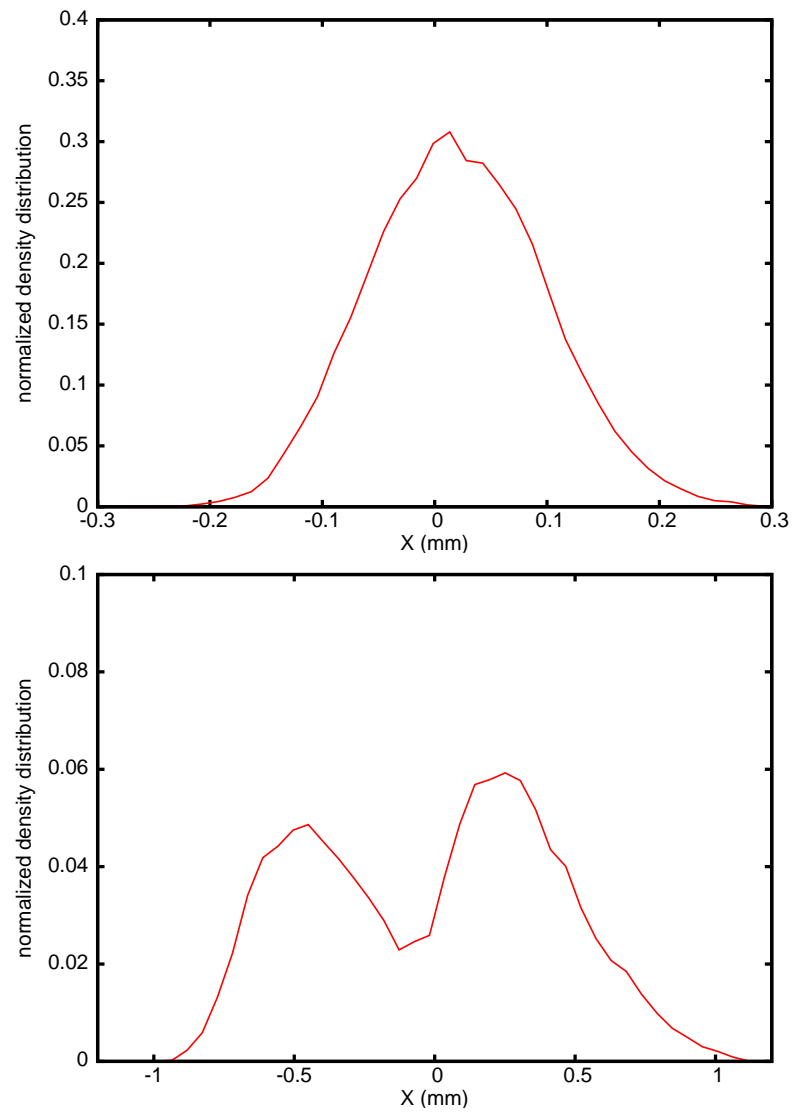

Fig. 5. Line density distribution at the image plane with initial 330 fs separation without (top) with (bottom) longitudinal magnification through the streak camera.

As a systematic comparison the two systems (with/without longitudinal magnification), we also compute the temporal modulation transfer function (TMTF) through the streak camera. The calculated TMTF as a function of the inverse of the initial temporal separation is given in Fig. 6. It is seen that using longitudinal temporal magnification has significantly improved the temporal resolution of the streak camera. 


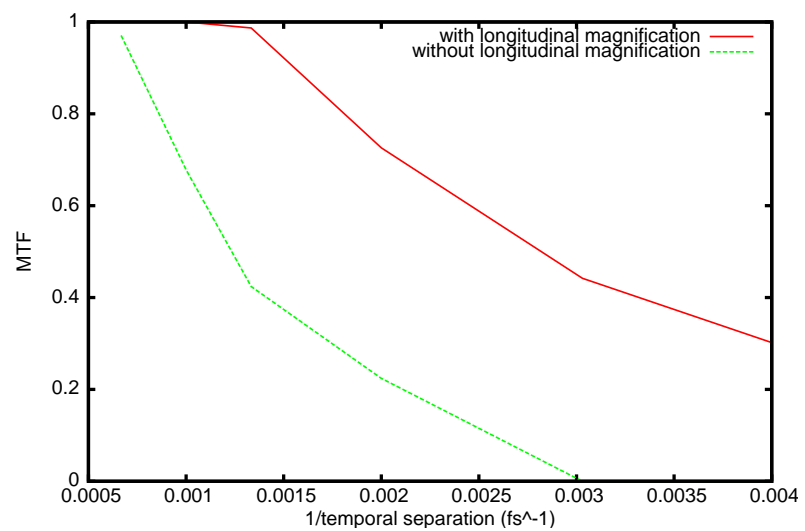

Fig. 6. Modulation transfer function as a function of temporal separation frequency with/without longitudinal magnification through the streak camera.

\section{Discussions}

In section 2 and 3, we presented the theory of longitudinal magnification and showed an example of temporal resolution improvement by ramping down accelerating field in a prototype streak camera. From Eq. 8, the final time difference of an electron passing through a time-dependent field depends both on the initial time difference and the initial energy spread of electron bunch. In practice, the second term can be made much smaller than the first term using a large ramping slope of the time-dependent field. The large ramping slope of the field can be achieved in a pulsed gap or in a rf cavity. The photo electrons passing through a time dependent field also obtain a correlated energy chirp, which could cause chromatic aberration of the beam through a magnetic focusing system. This chromatic effect can be corrected after the deflection by using another time-dependent field or by magnetic elements such as sextupole and dipole. On the other hand, such an energy chirp might even be taken advantage to generate transverse image by passing through a bending magnet. Due to the longitudinal magnification, the total electron bunch length will increase before the deflection plate. The corresponding transverse beam size will also increase after vertical deflection. This puts stronger requirement for the transverse magnetic focusing system due to nonlinear geometric aberration. This aberration can be avoided by using a focusing element with sufficiently large dynamic aperture. Through the longitudinal magnification, the photo electron bunch becomes longer and larger after deflection, this also helps to mitigate the space-charge effects since the Coulomb interactions decrease with increasing distance among electrons. It will be especially useful for single shot measurement of high intensity photon signal. 


\section{Acknowledgements}

We would like to thank Drs. H. Padmore and W. Wan for discussions. This work was supported by the of Science, U. S. Department of Energy, under Contract No. DE-AC02-05CH11231. We have used computing resources at

National Energy Research Scientific Computing Center, a DOE office of science user facity at Lawrence Berkeley National Laboratory.

\section{References}

[1] Z. Chang, A. Rundquist, J. Zhou, M. M. Murnane, H. C. Kapteyn, X. Liu, B. Shan, J. Liu, L. Niu, M. Gong, X. Zhang, Appl. Phys. Lett. 69 133, 1996.

[2] P. Gallant, P. Forget, F. Dorchies, Z. Jiang, J. C. Kieffer, P. A. Jaanimagi, J. C. Rebuffie, C. Goulmy, J. F. Pelletier, and M. Sutton, Rev. Sci. Instrum. 71 3627, 2000 .

[3] G. A. Naylor, K. Scheidt, J. Larsson, M. Wulff, and J. M. Filhol, Meas. Sci. Technol. 12 1858, 2001.

[4] K. Kinoshita, Y. Inagaki, Y. Ishhara, S. Abe, and M. Nishizawa, Jpn. J. Appl. Phys. 41 389, 2002.

[5] J. Liu, J. Wang, B. Shan, C. Wang, Z. Chang, Appl. Phys. Lett. 82 3553, 2003.

[6] J. Feng, W. Wan, J. Qiang, A. Bartelt, A. Comin, A. Scholl, J.Byrd, R. Falcone, G.Huang, A. MacPhee , J.Nasiatka, K. Opachich, D. Weinstein, T. Young and H.A.Padmore, "An Ultra-Fast X-Ray Streak Camera for the Study of Magnetication Dynamics," Proceedings of SPIE 5920, (2005) 592009.

[7] M. M. Murnane, H. C. Kapteyn, and R. W. Falcone, Appl. Phys. Lett. 56 1948, 1990.

[8] C. Belzile, J. C. Kieffer, C. Y. Cote, T. Oksenhendler, and D. Kaplan, Rev. Sc. Instrum. 73 1617, 2002.

[9] B. L. Henke, J. A. Smith, D. T. Attwood, J. Appli. Phys. 48 1852, 1977. 